DOI: $10.1002 /(($ please add manuscript number $))$

Article type: Communication

\title{
Experimental demonstration of metasurface-based ultrathin carpet cloaks for millimetre waves
}

Bakhtiyar Orazbayev, Nasim Mohammadi Estakhri, Andrea Alù*, Miguel Beruete*

B. Orazbayev, Dr. M. Beruete

Antennas Group-TERALAB

Universidad Pública de Navarra

Campus Arrosadía, 31006 Pamplona, Spain

E-mail: miguel.beruete@ unavarra.es

Dr. M. Beruete

Institute of Smart Cities

Public University of Navarra

31006 Pamplona, Spain

N. Mohammadi Estakhri, Dr. A. Alù

Department of Electrical and Computer Engineering

The University of Texas at Austin

Austin, Texas 78712, USA

E-mail: $\underline{\text { alu@mail.utexas.edu }}$

Keywords: invisibility cloaks; metasurfaces; full polarization

The possibility of making large objects undetectable has motivated significant research activities in the past decade. In its ideal operation, an electromagnetic cloak should be able to conceal an object within a wide operation bandwidth and a broad angular range, independent of the polarization of light. Although the first invisibility cloaks ${ }^{[1]}$ did not fulfill these constraints, were bulky and targeted mostly the lower-frequency spectrum, these seminal research activities have inspired several follow-up experimental demonstrations, including visible light cloaks. ${ }^{[2-4]}$ In this paper, we report the design and experimental demonstration of a metasurface carpet cloak working in the millimetre-wave range, independent of polarization, and with a relatively wide frequency and angular width response. We demonstrate that this ultrathin $\lambda_{0} / 19$ carpet cloak, based on a gradient metasurface design, reduces the scattering from 


\section{WILEY-VCH}

a $1.1 \lambda_{0}$ object and opens interesting perspectives for the use of cloaks and metasurfaces in millimetre-wave and terahertz applications.

To date, most invisibility cloaks have been designed by applying either the transformation optics technique $(\mathrm{TO})^{[5,6]}$ or the scattering cancellation technique. ${ }^{[7,8]}$ In TO cloaks, the concealed region is enclosed by an anisotropic cover that bends the impinging light beam around it, making the object effectively invisible. These cloaks are usually quite thick, suffer from losses, and their experimental realization is challenging. In turn, the scattering cancellation technique provides simpler designs with a more robust performance, and, in case of the mantle cloak based on patterned metasurface, these cloaks can be extremely thin..$^{[9,10]}$ However, this technique is also limited by the size of the cloaked objects ${ }^{[11]}$ and in its simplest form can be only applied to objects with sizes comparable to wavelength. Due to the fact that the number of the scattering harmonics and therefore the required number of cloaking layers increases with the size of the object, for larger objects the design becomes cumbersome and leads to similar complexities as the TO approach. The challenging requirements on the electromagnetic parameters imposed by TO can be relaxed when designing carpet cloaks ${ }^{[12]}$ based on the quasiconformal mapping technique. ${ }^{[12,13]}$ In this case cloaking is achieved by tailoring the material properties around the object to compress the effective volume of the object into an infinitesimally thin sheet over a ground plane. This transformation can be achieved with nonmagnetic materials with an inhomogeneous permittivity. Low refractive index gradient materials have been also explored to improve the impedance matching with free-space. ${ }^{[14]}$

Recently metasurfaces, i.e., artificially engineered ultrathin surfaces, have attracted significant interest, as they provide a powerful way of controlling the electromagnetic wave by manipulating the local amplitude and phase of the field. ${ }^{[15-17]}$ As a result, a different approach to cloaking has been recently introduced based on gradient metasurfaces. ${ }^{[18-21]}$ The cloaking effect is achieved by manipulating the phase of the reflected wave along the boundary of the 


\section{WILEY-VCH}

concealed volume. In this approach, the cloak is composed of an ultrathin gradient metasurface, artificially engineered with subwavelength elements that are tailored to control the phase of the reflected wave and restore the wavefront scattered from the object, emulating a flat ground plane. A metasurface cloak based on this principle has been experimentally demonstrated in optics, ${ }^{[22]}$ showing interesting perspectives for this cloaking technique. Moreover, a recent experimental work on metasurface-based cloaks in the low-band microwave range shows the possibility of restoring not only the amplitudes and phases, but also the initial polarization ${ }^{[23]}$. Although for higher frequencies the fabrication is more challenging, due to the required smaller sizes of the metasurface elements, this method can also find applications in other frequency ranges, such as the emergent millimetre-wave and $\mathrm{THz}$ bands. A polarization independent metasurface based cloaking device in these frequency ranges may be promising in a variety of sectors such as communication, security, military, space and biomedical applications. ${ }^{[24,25]}$ Moreover, as mentioned above, a response independent of polarization is critical for real-life applications given that the polarization of the incident wave is usually unknown. In our previous work, ${ }^{[20]}$ we numerically analysed a terahertz carpet cloak based on a gradient metasurface made of closed ring resonators. We demonstrated that such cloak is able to restore the wavefront and phase of the reflected beam, reducing the unwanted scattering from a bump. Here, we improve this theoretical design and experimentally verify a cloaking prototype operating at 80 $\mathrm{GHz}$, demonstrating scattering cross-section reduction from a metallic bump over a ground plane within relatively wide range of angles and frequencies for both transverse electric (TE) and transverse magnetic (TM) incident polarizations.

Figure 1 shows the metasurface cloak considered in this work. The object to be concealed is a triangular bump with height $4.1 \mathrm{~mm}\left(1.1 \lambda_{0}\right.$ at $\left.80 \mathrm{GHz}\right)$ and long side $40 \mathrm{~mm}\left(10.7 \lambda_{0}\right)$. $\lambda_{0}$ denotes the center wavelength in free-space. The restoration of the scattered wavefront is achieved by compensating the phase difference $\delta$ between the waves reflected from the ground 


\section{WILEY-VCH}

plane and the boundary of the concealed object, calculated at each point of the bump's edge following the method discussed in [18-21]

$\delta=\pi-2 k_{0} h \cos \theta$

where $k_{0}$ is the free-space wave vector at the operation frequency, $h$ is the height of the point from the ground plane, and $\theta$ is the angle of the incident wave with respect to the ground plane normal. As shown in the bottom left inset of Figure 1a, our metasurface is composed of double coaxial metallic rings with total metal thickness $0.8 \mu \mathrm{m}$, period of $400 \mu \mathrm{m}\left(\lambda_{0} / 9\right)$ and gap between rings of $10 \mu \mathrm{m}$, placed on a high resistivity silicon substrate $(\rho>10000 \Omega \cdot \mathrm{m})$ with thickness of $200 \mu \mathrm{m}\left(\lambda_{0} / 19\right)$ (see Supporting Information for more details). This geometry was chosen to make the metasurface insensitive to the polarization of the incident wave. ${ }^{[19]}$ The response of each unit cell was tuned by changing the radii of the rings. The amplitude and phase curves of the reflection coefficient as a function of the ring radius are shown in the bottom right inset of Figure 1a. From this figure, it can been seen that for small incidence angles the phase response is almost identical for both polarizations, whereas for large angles the phase response for TM polarization deviates from TE polarization. In principle, the polarization dependence can affect the performance of the cloak with large corner angles, which may be circumvented by increasing the footprint of the cloaking layer.

Due to availability and mechanical restrictions, the silicon thickness is different from the one used in ref. $20(165 \mu \mathrm{m})$. Therefore, an additional optimization of the cloaking metasurface was performed for the new silicon thickness $(200 \mu \mathrm{m})$, resulting in a new optimal cloaking metasurface configuration. Metasurface layers were fabricated by using a photolithography process followed by a lift-off technique to print the metallic rings on the silicon substrate (detailed fabrication specifications can be found in the Supporting Information). A photograph of the fully cloaked structure with the fabricated metasurface covering the triangular bump is presented in Figure 1b, along with a microscope picture showing details of the metallic rings. 


\section{WILEY-VCH}

To evaluate the cloaking performance, we used two different experimental setups (top right inset of Figure 1b): based on an ABmm vector network analyser (VNA) (setup 1) and an Agilent E3861C VNA (setup 2). In both setups the reflection coefficient at different angles was recorded in the range 75-95 GHz, using two corrugated high gain horn antennas as transmitter and receiver. The distance from the transmitting horn antenna to the sample was sufficient to ensure a uniform illumination of the sample, at the same time avoiding diffraction from the metallic plane edges (Complete details of both setups are given in the Supporting Information). In the first study, TE polarization with incidence angle $\theta=45^{\circ}$ was used. In this configuration, we measured three samples: bare ground plane, metallic bump on the ground plane, and bump, when it is cloaked with the designed gradient metasurface.

The color maps of the reflected electric field distribution as a function of frequency and azimuthal angle $\varphi$ are shown in Figure 2a-c, for all three cases. As expected, the electrically large bare bump strongly scatters the electric field, resulting in two additional high peaks on both sides of the main specular reflection for all measured frequencies. When the cloak is applied, these peaks are significantly suppressed (almost $-20 \mathrm{~dB}$ for some angles, see Figure. $2 \mathrm{~d}, \mathrm{~h}$, where the experimental and numerical radiation patterns are shown for $f_{c}=86 \mathrm{GHz}$ and 83.5 GHz respectively), confirming the scattering reduction. These results are compared with numerical simulations done with CST Microwave Studio in Figure 2e-g (details about the numerical simulation setup are given in the Supporting Information). As observed, measurements and simulations are in good agreement. The experiment presents a slight blue$\operatorname{shift}\left(f_{c} \approx 86 \mathrm{GHz}\right)$ with respect to the design $\left(f_{c}=80 \mathrm{GHz}\right)$ and also simulation frequency $\left(f_{c}=\right.$ 83.5 GHz). We attribute this shift to multiple factors, such as deviations in the gap between the rings and the thickness of the fabricated metallic rings, due to the tolerances in the fabrication process. From additional simulations, shown in the Supplementary Figure S1, it can be seen that the central frequency $f_{c}$ blue-shifts as the metal thickness is increased. Comparing the 


\section{WILEY-VCH}

simulation results with those in Ref. 20 (that correspond to a substrate height of $165 \mu \mathrm{m}$ ) it is clear that, even though the substrate thickness and therefore the optimal cloaking metasurface are not the same, the present cloak is still able to significantly reduce the scattering across almost the same frequency range. The most important difference is that the main beam shape is not ideally recovered (see Supplementary Figure S2).

Next, we analyze the cloaking performance under TM-polarization. The numerical and measured radiation patterns for all three cases are shown in Figure 3. In a similar way as for TE incidence, the cloak is able to reduce the scattering from the metallic triangular bump in the analogous frequency range. Moreover, we observe excellent agreement between measurements and simulation results (Figure 3e-g). The overall cloaking performance is however slightly degraded in comparison with the TE case (see Figure 3d, h). In order to quantitatively assess this difference in performance we calculated the total scattering gain for $\theta=45^{\circ}$ and both polarizations, defined as the ratio between the total radar cross section (RCS) of the cloaked and bare bump, using the procedure described in the Supporting Information. The results are shown in Figure 4a. A scattering reduction is clearly observed for both TE and TM polarizations, with a minimum total scattering gain around $86 \mathrm{GHz}$. In the TE case, we acheive $-6.5 \mathrm{~dB}$, whereas under TM incidence the minimum is less pronounced (around $-4.5 \mathrm{~dB}$ ), demonstrating slightly worse performance for this polarization. This degradation of performance can be due to the fact that in TM-polarization the design is more sensitive to the substrate thickness and the non-uniformity of the structure (which manifests itself at large incidence angles). Moreover, due to the relatively large corner angle $\left(20^{\circ}\right)$, in TM-polarization the phase response of the metasurface's unit cell differs from TE-polarization, as it was shown in the inset of Fig.1(a). In order to achieve analogous performance in TM polarization one could use a more complicated structure, for instance, a pyramidal shape, which restores the phase distribution on the metasurface more accurately. ${ }^{[21,23]}$ Additional simulations (not shown here) show that for the 


\section{WILEY-VCH}

substrate thickness $(165 \mu \mathrm{m})$ used in ref. 20 the cloaking performance for both polarizations is in better agreement for smaller incidence angles $\left(\theta<45^{\circ}\right)$.

In order to quantitatively evaluate the bandwidth and angular span of the fabricated cloak, we measured the RCS gain and calculated the total scattering gain $G(f, \theta)$ for incidence angles ranging from $25^{\circ}$ to $55^{\circ}$, focusing in this case on TE polarization. For this purpose we used setup 2 and the azimuthal distribution of the reflection coefficient for all three cases mentioned above is obtained. This information was converted to RCS using the radar range equation $^{[26]}$ (for details of the extraction procedure see Supporting Information). In Figure $4 \mathrm{~b}$ the experimental total scattering gain is shown as a function of frequency and incidence angle $\theta$. In good agreement with the results obtained with setup 1, the RCS of the cloaked bump is suppressed by over $6 \mathrm{~dB}$ around $f_{c}=86 \mathrm{GHz}$ and $\theta=45^{\circ}$. As shown in Figure $4 \mathrm{~b}$, the cloak demonstrates a $5 \mathrm{~dB}$ RCS suppression within a relatively large fractional bandwidth ( $\sim 8 \%)$ and angular $\operatorname{span}\left(\sim 15^{\circ}\right)$.

The presented results show that an ultrathin metasurface cloak $\left(\lambda_{0} / 19\right)$ operating at millimetre-wave frequencies can supress the scattering from a moderately large bump over a ground plane, independent of the incident polarization. The measured prototype demonstrates a relatively wide frequency range with fractional bandwidth of $8 \%$ and angular span of $15^{\circ}$. Although the triangular shape of the bump makes the cloak more sensitive to imperfections of the metasurface (especially on top of the structure), it greatly facilitates the fabrication process, making it more feasible for a practical implementation. This work also highlights the possibility of building polarization-independent practical cloaks for macroscopic objects that may work for unknown polarization and over a relatively wide angular and frequency ranges, and may be integrated in millimetre-wave applications, such as radar automotive systems or ultrahigh speed telecommunication systems. More broadly, since this metasurface cloak can be scaled up to the THz band, our work shows how gradient metasurfaces may make a significant impact to control 


\section{WILEY-VCH}

and tailor $\mathrm{THz}$ radiation and wave propagation, in a frequency range for which ultrathin components and devices are scarcely available and inefficient, and for which deeply subwavelength control may be routinely achieved with conventional fabrication processes.

\section{Supporting Information}

Supporting Information is available online from the Wiley Online Library or from the author.

\section{Acknowledgements}

Authors are grateful to Adrian Gomez Torrent for help in fabrication of samples. This work was founded by the Spanish Ministerio de Economía y Competitividad with project TEC2014-51902-C2-2-R. M.B. acknowledges funding by the Spanish Ministerio de Economía y Competitividad with contract RYC-2011- 08221. N.M.E. and A.A. have been supported by the NSF CAREER Award No. ECCS-0953311 and the AFOSR Grant No. FA9550-13-1-0204.

Received: ((will be filled in by the editorial staff))

Revised: ((will be filled in by the editorial staff)) Published online: ((will be filled in by the editorial staff))

[1] D. Schurig, J. J. Mock, B. J. Justice, S. A. Cummer, J. B. Pendry, A. F. Starr, D. R. Smith, Science. 2006, 314, 977.

[2] J. Valentine, J. Li, T. Zentgraf, G. Bartal, X. Zhang, Nat. Mater. 2009, 8, 568.

[3] L. H. Gabrielli, J. Cardenas, C. B. Poitras, M. Lipson, Nat. Photonics 2009, 3, 12.

[4] M. Gharghi, C. Gladden, T. Zentgraf, Y. Liu, X. Yin, J. Valentine, X. Zhang, Nano Lett. 2011, 11, 2825.

[5] U. Leonhardt, Science. 2006, 312, 1777.

[6] J. B. Pendry, Science. 2006, 312, 1780.

[7] A. Alù, N. Engheta, Phys. Rev. E 2005, 72, 16623.

[8] D. Rainwater, A. Kerkhoff, K. Melin, J. C. Soric, G. Moreno, A. Alù, New J. Phys. 2012, 14, 13054.

[9] A. Alù, Phys. Rev. B 2009, 80, 245115.

[10] P.-Y. Chen, A. Alù, Phys. Rev. B 2011, 84, 205110. 


\section{WILEY-VCH}

[11] F. Monticone, A. Alù, Phys. Rev. X 2013, 3, 41005.

[12] J. Li, J. B. Pendry, Phys. Rev. Lett. 2008, 101, 203901.

[13] R. Liu, C. Ji, J. J. Mock, J. Y. Chin, T. J. Cui, D. R. Smith, Science. 2009, 323, 366.

[14] H. F. Ma, W. X. Jiang, X. M. Yang, X. Y. Zhou, T. J. Cui, Opt. Express 2009, 17, 19947.

[15] N. Yu, P. Genevet, M. A. Kats, F. Aieta, J.-P. Tetienne, F. Capasso, Z. Gaburro, Science. 2011, 334, 333.

[16] A. V. Kildishev, A. Boltasseva, V. M. Shalaev, Science. 2013, 339, 1232009.

[17] N. Yu, F. Capasso, Nat. Mater. 2014, 13, 139.

[18] J. Zhang, Z. Lei Mei, W. Ru Zhang, F. Yang, T. Jun Cui, Appl. Phys. Lett. 2013, 103, 151115.

[19] N. Mohammadi Estakhri, A. Alù, IEEE Antennas Wirel. Propag. Lett. 2014, 13, 1775.

[20] B. Orazbayev, N. Mohammadi Estakhri, M. Beruete, A. Alù, Phys. Rev. B 2015, 91, 195444.

[21] N. Mohammadi Estakhri, C. Argyropoulos, A. Alù, Philos. Trans. R. Soc. A Math. Phys. Eng. Sci. 2015, 373, 20140351.

[22] X. Ni, Z. J. Wong, M. Mrejen, Y. Wang, X. Zhang, Science. 2015, 349, 1310.

[23] Y. Yang, L. Jing, B. Zheng, R. Hao, W. Yin, E. Li, C. M. Soukoulis, H. Chen, Adv. Mater. 2016, DOI 10.1002/adma.201600625.

[24] P. H. Siegel, IEEE Trans. Microw. Theory Tech. 2002, 50, 910.

[25] M. Tonouchi, Nat. Photonics 2007, 1, 97.

[26] C. A. Balanis, Antenna Theory: Analysis and Design, Wiley, 2015. 


\section{WILEY-VCH}
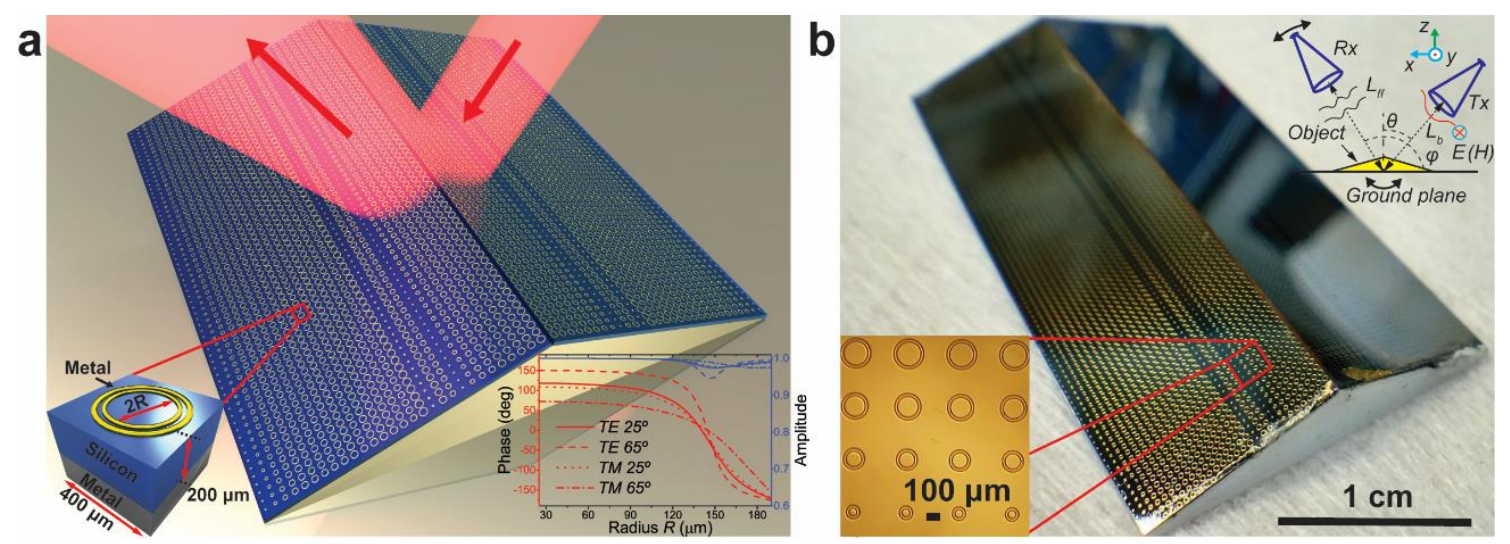

Figure 1. Schematic illustration and photographs of the fabricated metasurface cloak. a) 3D view of the cloak metasurface composed of an array of double coaxial metallic ring. Left inset: detail of a metasurface element with representative parameters. Right inset: amplitude and phase response of the unit cell as a function of the ring radius for both polarizations and different incidence angle. b) Photograph of the fabricated cloak. Left inset: microscope picture showing a detail of the fabricated rings. Right inset: scheme of experimental set-up.

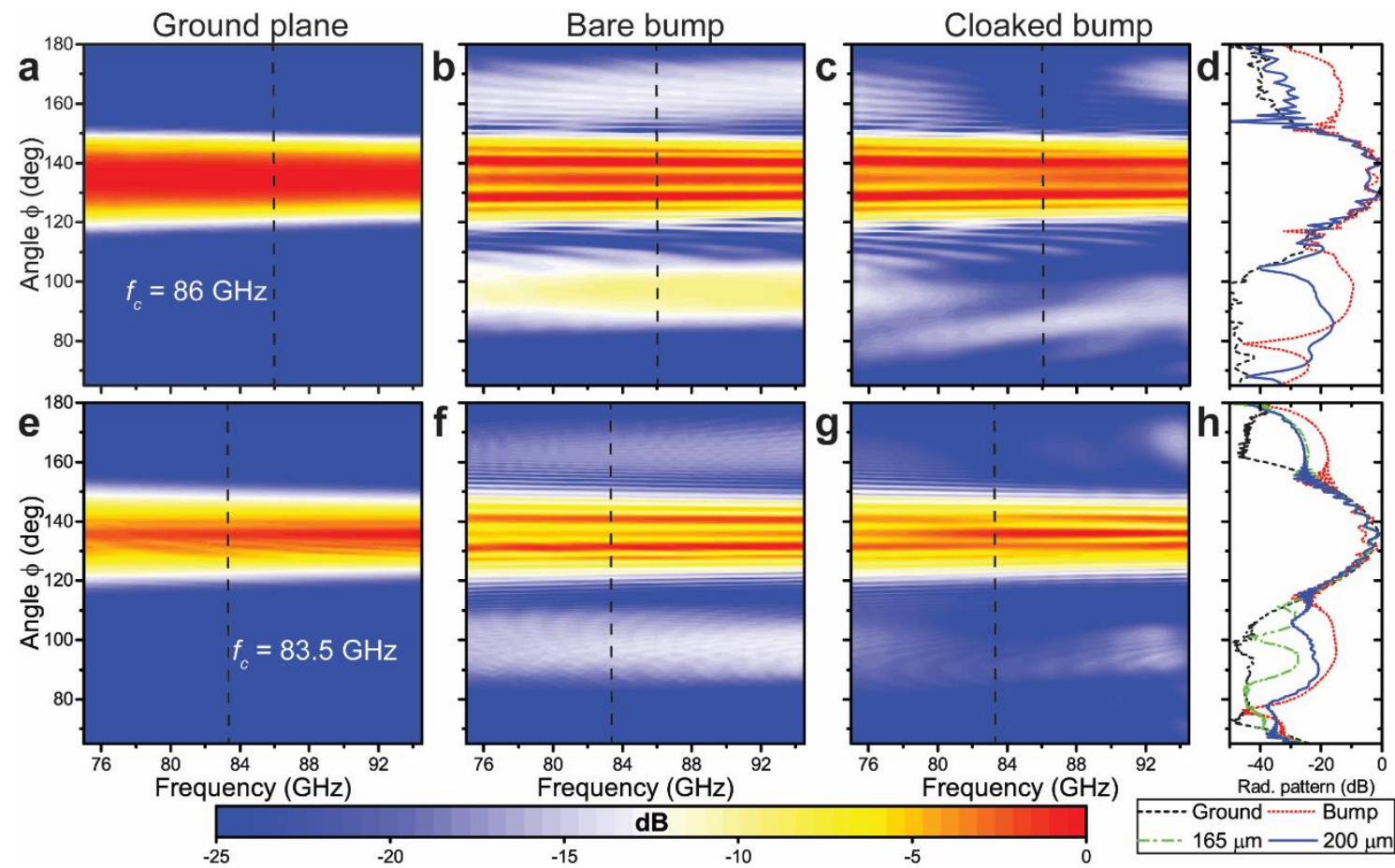

Figure 2. Radiation pattern for ground plane, bare bump and cloaked bump at the optimum incidence angle $\theta=45^{\circ}$ and TE-polarization. a-c) Experimental results. d) Experimental 


\section{WILEY-VCH}

radiation patterns for all three cases at $f_{\mathrm{c}}=86 \mathrm{GHz}$. e-g) Simulation results. h) Numerical radiation patterns at frequency $f_{c}=83.5 \mathrm{GHz}$.

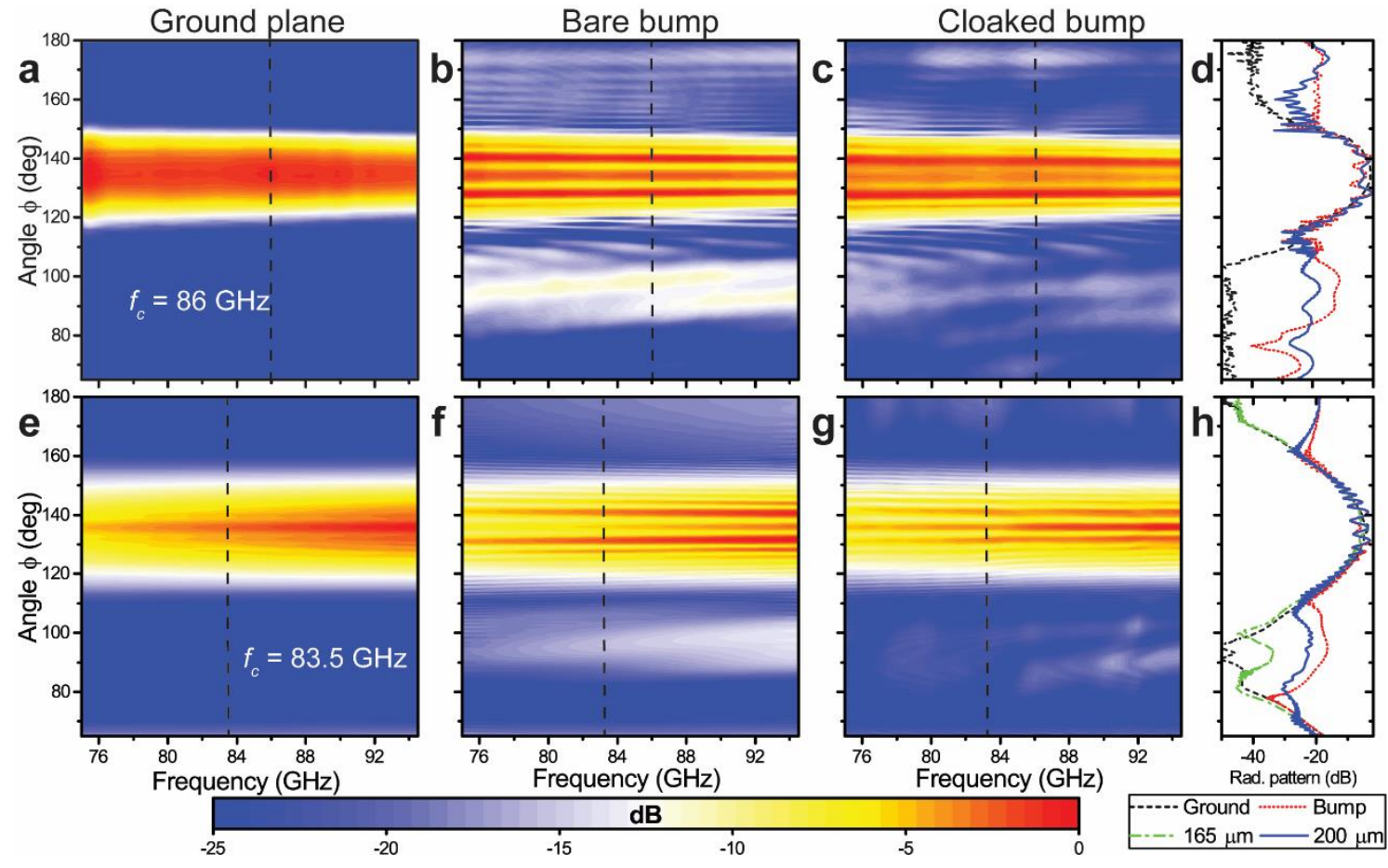

Figure 3. Radiation pattern for ground plane, bare bump and a cloaked bump at the optimum incidence angle $\theta=45^{\circ}$ and TM-polarization. a-c) Experimental results. d) Experimental radiation patterns for all three cases at $f_{\mathrm{c}}=86 \mathrm{GHz}$. e-g) Simulation results. h) Numerical radiation patterns at frequency $f_{c}=83.5 \mathrm{GHz}$.
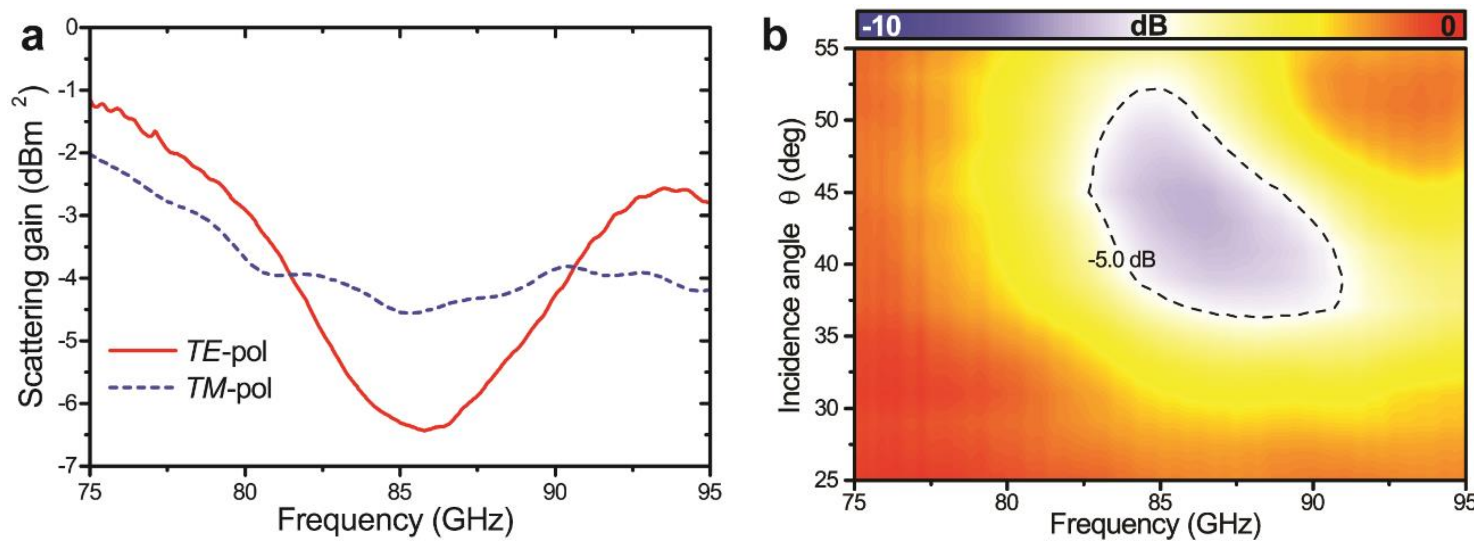


\section{WILEY-VCH}

Figure 4. Experimental total scattering gain. a) Experimentally measured total scattering gain at the optimum incidence angle $\theta=45^{\circ}$ for the TE-polarization (solid red line) and for the TMpolarization (dashed blue line). b) Experimental total scattering gain vs incident angle and frequency for TE-polarization. 